\title{
FINDING MULTIPLE SOLUTIONS OF MULTIMODAL OPTIMIZATION USING SPIRAL OPTIMIZATION ALGORITHM WITH CLUSTERING
}

\author{
Kuntjoro Adji Sidarto, Adhe Kania and Novriana Sumarti
}

\author{
Institut Teknologi Bandung \\ Department of Mathematics \\ Jl. Ganesha 10, Bandung 40132 \\ Indonesia \\ sidarto@math.itb.ac.id, adhe.kania@math.itb.ac.id, novriana@math.itb.ac.id
}

\begin{abstract}
A Multimodal optimization is one of the interesting problems in optimization which arises frequently in a wide range of engineering and practical applications. The goal of this problem is to find all of optimum solutions in a single run. Some algorithms fail to find all solutions that have been proven their existence analytically. In our paper [1], a method is proposed to find the roots of a system of non-linear equations using a clustering technique that combine with Spiral Optimization algorithm and Sobol sequence of points. An interesting benefit using this method is that the same inputs will give the same results. Most of the time this does not happen in meta-heuristic algorithms using random factors. Now the method is modified to find solutions of multimodal optimization problems. Generally in an optimization problem, the differential form of the objective function is needed. In this paper, the proposed method is to find optimum points of general multimodal functions that its differential form is not required. Several problems with benchmark functions have been examined using our method and they give good result.
\end{abstract}

Keywords: Multimodal optimization, multiple optimal solutions, spiral optimization algorithm, clustering, Sobol sequence of points.

\section{Introduction}

Many problems in the real world can be modelled as optimization problems. Some variation of functions that increase their complexity may be needed as the objective function of multimodal optimization problems. The function can have numerous global and local maximum values. Unfortunately, most of optimization methods only give a single solution at one run. It could give other solutions if $t$ he in itial points are ch anged. To gu ess the in itial points is $n$ ot an easy task either. Recently, metaheuristic methods inspired by nature can get results without dependence with initial points, but it must be run in a number of times to obtain numerous solutions.

There are many approaches in solving the multimodal optimization problems. In [2], the Feature Se lection (FSel) problem that reduce the pattern dimensionality in data pre-processing is b eing solved using Multimodal Optimization (MO) techniques. The FSel problem is a h igh-dimensional optimization problem in the nature and thus needs a solver with high exploration power. MO's so lution conservation capability can find its multiple suitable solutions in a sin gle run.

In [3], the advanced multimodal Particle Swarm Optimization (PSO) algorithms adopted into the species-based PSO (SPSO), the fitness Euclidean-distance ratio based PSO (FER-PSO), the ring to pology based PSO and the Euclidean distance-based lo cally in formed $\mathrm{p}$ article swarm (LIPS) optimizer. Th ese algo rithms are ap plied to a m ultimodal buckling maximization problem of composite panels and identify simultaneously not only the first-best-fitness solutions (the gl obal optima) but al so the seco nd-best-fitness s olutions (t he gl obal sub-optima) to this buc kling optimization design in a single optimization process.

In [4], Differential Evolution (DE) algorithm was successfully extended with multi-population and multi-resolution approach to locate multiple optima of a multimodal function. In this method, the beneficial of DE, an efficient global optimization method, was com bined with $\mathrm{m}$ ulti-population in exploration process and $\mathrm{m}$ ulti-resolution to control initialization step. One and two dimensional benchmark functions is applied to prove the effectivity of this method.

In [5], the au thors proposed a fram ework, called MOMCA (multimodal optimization for model calibration), which is based on niching genetic algorithms. It provides a set of alternative calibration solutions, which is useful to analyse the parameters, model's response, and doing sensitivity analysis. The core component of MOMCA is its niching genetic algorithm, able to reach va rious optima in multimodal optimization proble ms by keeping the nece ssary diversity. The framework is successfully applied to a biological growth model and a managerial model to improve brand equity.

In [6], the stochastic multimodal optimization problems and stochastic resource allocation problem are being solved using a hybrid ap proach com prising p article swarm o ptimization (PSO), con striction fact or PSO (CPSO), and elite group optimal computing budget allocation (EGOCBA). CPSO or PSO is app lied to determine the correct direction in the design s pace, and EGOCBA is adop ted to allocate the appropria te num ber of sam ples to ea ch altern ative and 
provide reliable evaluations and id entifications to rank particles in the CPSO or PSO procedure. The approach has efficiently and effectively derived multiple optima of sets in a multimodal class and stochastic environment.

In this paper, we propose a new clustering method to get all of optimum values, both local and global, of multimodal function in a s ingle run. We use a nature-inspired metaheuristics method based on the spiral optimization, or in short SPO (Spiral Optimization) as in [7,8]. A stability analysis at the dynamic center of the S PO model has been st udied in [8], and it suggests a method for setting the convergence rate parameter. The method has attracted many modeller due to the simplicity to program, short computing time, easy to implement, and the possibility to apply to various applications [9]. O ne of the im provement pro posed in [9], i s LAS DA (linear ad aptive sp iral d ynamics alg orithm), wh ich is an improved version of SDA (adaptive spiral dynamics algorithm), where the spiral radius and a ngular displacement are dynamically varied by employing novel mathematical equation based on a linear function. This function establishes a relationship among fitness value, spiral radius and angular displacement. The proposed algorithm is tested with various types of multimodal and u nimodal benchmark functions. The results show a better performance in finding an optimal solution for the benchmark functions in comparison with other SDA approaches. The paper [1] and this paper propose an improvement by implementing the clustering technique. There is also a new technique in this paper in dealing with more general multimodal optimization problems, where their differential form of the objective functions is not existing or difficult to obtain. Some benchmark multimodal functions from [10] have been examined, and the proposed method has successfully found numerous possible solutions for those functions.

In the next section a revi ew of S piral Optimization Algorithm and the proposed clustering technique for 1 ocating multiple solutions are given. Section 3 presents numerical experiments on s ome benchmark functions. Finally some conclusions are given in Section 4.

\section{Methodology}

\subsection{Spiral Optimization Algorithm}

Consider an optimization problem of the form

$$
\text { maximize } F(\mathbf{x})
$$

with $\mathbf{x}=\left(x_{1}, x_{2}, \ldots, x_{n}\right)^{T} \in D=\left[a_{1}, b_{1}\right] \times\left[a_{2}, b_{2}\right] \times \cdots \times\left[a_{n}, b_{n}\right] \subset \mathbb{R}^{n}$ nd $F: D \rightarrow \mathbb{R}$. Here $F$ has multiple peaks in $D$.

SPO algorithm is a metaheuristic method for continuous optimization problems, developed by Tamura and Yasuda [7], base $d$ on the analogy of spiral phenomena in nature. Firstly we review 2- dimensional SPO before discu ss $n$ dimensional SPO, as some results are just extension of 2-dimensional SPO.

The $\mathrm{m}$ ultiplication of a vector $\mathbf{x} \in \mathbb{R}^{2}$ in the plane by $\mathrm{m}$

atrix $S_{2}(r, \theta)=\operatorname{diag}_{2}(r, r) \cdot R^{(2)} \mathrm{w}$ here $\operatorname{diag}_{2}(r, r)=\left(\begin{array}{ll}r & 0 \\ 0 & r\end{array}\right)$ and $R^{(2)}=\left(\begin{array}{cc}\cos \theta & -\sin \theta \\ \sin \theta & \cos \theta\end{array}\right)$ is a ro tation matrix in the p lane, gives the ge ometric effect by rotating $\mathbf{x}$ ant iclockwise through the a ngle $\theta(0<\theta \leq 2 \pi)$, and s hortening the vector $R^{(2)} \mathbf{x}$ by a fact or of $r$ fo $\mathrm{r}$ $0<r<1$. Hence the equation

$$
\mathbf{x}_{k+1}=S_{2}(r, \theta) \mathbf{x}_{k} \quad \text { where } \quad \mathbf{x}_{k}=\mathbf{x}(k)=\left(x_{1}(k), x_{2}(k)\right)^{T} \quad k=0,1,2, \ldots
$$

describes the transformation of an initial point $\mathbf{x}_{0} \in \mathbb{R}^{(2)}$ repeatedly by $\mathbf{x} \mapsto S_{2}(r, \theta) \mathbf{x}$. The graph of the sequence of points $\mathbf{x}_{0}, \mathbf{x}_{1}, \mathbf{x}_{2}, \ldots$ in the plane will $\mathrm{p}$ roduce a traj ectory of $\mathrm{sp}$ iral form. So, it see $\mathrm{ms}$ th at the point $\mathbf{x}_{0}$ goes spira 1 inward an $\mathrm{d}$ toward the orig in for $0<r<1$. The spi ral model described earl ier $\mathrm{h}$ as a center at the ori gin. It can be extended to have center at an arbitrary point $\mathbf{x}^{*}$ by translating the origin toward the point $\mathbf{x}^{*}$ in Eq.(1) :

$$
\mathbf{x}_{k+1}-\mathbf{x}^{*}=S_{2}(r, \theta)\left(\mathbf{x}_{k}-\mathbf{x}^{*}\right) \text { such that } \mathbf{x}_{k+1}=S_{2}(r, \theta) \mathbf{x}_{k}-\left(S_{2}(r, \theta)-I_{2}\right) \mathbf{x}^{*} \quad k=0,1,2, \ldots
$$

The iterated point $\mathbf{u}_{k}=\mathbf{u}(k)=\mathbf{x}_{k}-\mathbf{x}^{*} \quad k=0,1,2, \ldots$ will move sp irally in ward and toward the orig in. Hen ce, the iterated point $\mathbf{x}_{0}$ will move spirally inward and toward the point $\mathbf{x}^{*}$ in the plane.

Let us consider the optimization problem

$$
\underset{\mathbf{x} \in \mathbb{R}^{2}}{\operatorname{maximize}} F(\mathbf{x}) \text { with } \mathbf{x}=\left(x_{1}, x_{2}\right)^{T} \in \mathbb{R}^{2}
$$

Based on the previous spiral model, the SPO algorithm in the plane is developed as follows.

Input : $m(\geq 2)$ the number of search points, $\theta(0<\theta \leq 2 \pi), r(0<r<1), k_{\max }$ maximum number of iteration

Process : 1. Randomly generate initial points $\mathbf{x}_{i}(0) \in \mathbb{R}^{2} i=1,2, \ldots, m$ in the feasible region.

2. Set $k=0$. 
3. Find $\mathbf{x}^{*}=\mathbf{x}_{i_{g}}(0)$ where $i_{g}=\arg \max F\left(x_{i}(0)\right) \quad i=1,2, \ldots, m$

4. Update $\mathbf{x}_{i}: \mathbf{x}_{i}(k+1)=S_{2}(r, \theta) \mathbf{x}_{k}-\left(S_{2}(r, \theta)-I_{2}\right) \mathbf{x}^{*} \quad k=0,1,2, \ldots, m$

5. Update $\mathbf{x}^{*}: \mathbf{x}^{*}=\mathbf{x}_{i_{g}}(k+1), \quad i_{g}=\arg \max F\left(x_{i}(k+1)\right) \quad i=1,2, \ldots, m$

6. If $k=k_{\max }$ then stop. Otherwise, set $k=k+1$ and return to step 4 .

Output : $\mathbf{x}^{*}$ as a maximum point of $F(\mathbf{x})$.

The exte nsion to $n$-dimensional S PO $\mathrm{m}$ ay be do ne with the help of rotation $\mathrm{m}$ atrix in $n$-dimensional which is performed in a sim ilar way as the 2- dimensional rotation taking two-dimensions at a tim e. Let $R_{i j}^{(n)}$ be $n \times n$ matrix with entries $r_{i i}=r_{j j}=\cos \theta, r_{i j}=-\sin \theta$, and $r_{j i}=\sin \theta$, and for other entries, $s \neq i, t \neq j, r_{s t}=\delta_{s t}$, where $\delta_{s t}=1$ if $s=t$ and $\delta_{s t}=0$ if $s \neq t$. Thus $R_{i j}^{(n)}$ resemble the identity matrix with exception for $i i, i j, j j$ and $j i$ positions. Multiplication of a vector by rotation matrix $R_{i j}^{(n)}$ only alter $i^{\text {th }}$ and $j^{\text {th }}$ components of the vector with no effect on other components. Hence, $R_{i j}^{(n)}$ acts as the plane rotation. Let $R^{(n)}$ be $n \times n$ matrix defined by

$$
R^{(n)}=\prod_{i=1}^{n-1}\left(\prod_{j=1}^{i} R_{n-i, n+1-j}^{(n)}\right)
$$

which is a co mposition of plane rotations matrix $R_{i j}^{(n)}$. The $n$-dimensional spiral m odel with center at the origin is formulated as $\mathbf{x}(k+1)=S_{n}(r, \theta) \mathbf{x}(k)$ where $S_{n}(r, \theta)=\operatorname{diag}_{n}(r, r, \ldots, r) \cdot R^{(n)}$ and the $n$-dimensional spiral m odel with center at $\mathbf{x}^{*}$ is formulated as : $\mathbf{x}(k+1)=S_{n}(r, \theta) \mathbf{x}(k)-\left(S_{n}(r, \theta)-I_{n}\right) \mathbf{x}^{*}$.

Let us consider the optimization problem

$$
\underset{\mathbf{x} \in \mathbb{R}^{n}}{\operatorname{maximize}} F(\mathbf{x}) \text { with } \mathbf{x}=\left(x_{1}, x_{2}, \ldots, x_{n}\right)^{T} \in \mathbb{R}^{n}
$$

The $n$-dimensional SPO algorithm is written below.

Input : $\quad m(\geq 2)$ the number of search points, $\theta(0<\theta \leq 2 \pi), r(0<r<1), k_{\max }$ maximum number of iteration

Process : 1. Randomly generate initial points $\mathbf{x}_{i}(0) \in \mathbb{R}^{n} i=1,2, \ldots, m$ in the feasible region.

2. Set $k=0$.

3. Find $\mathbf{x}^{*}=\mathbf{x}_{i_{g}}(0)$ where $i_{g}=\arg \max F\left(x_{i}(0)\right) \quad i=1,2, \ldots, m$

4. Update $\mathbf{x}_{i}: \mathbf{x}_{i}(k+1)=S_{2}(r, \theta) \mathbf{x}_{k}-\left(S_{2}(r, \theta)-I_{2}\right) \mathbf{x}^{*} \quad k=0,1,2, \ldots, m$

5. Update $\mathbf{x}^{*}: \mathbf{x}^{*}=\mathbf{x}_{i_{g}}(k+1), \quad i_{g}=\underset{i}{\arg \max } F\left(x_{i}(k+1)\right) \quad i=1,2, \ldots, m$

6. If $k=k_{\max }$ then stop. Otherwise, set $k=k+1$ and return to step 4 .

Output : $\mathbf{x}^{*}$ as a maximum point of $F(\mathbf{x})$.

\subsection{A Clustering Technique for Locating Multiple Solution Points}

In general, problem (1) $\mathrm{m}$ ay have $\mathrm{m}$ any solutio ns corresponding to 1 ocal minimum and global maximum points of $F(\mathbf{x})$. A sin gle run of S PO algorithm can only obtain a sin gle global maximum point. In an attempt to locate other solutions in a single run, a clustering technique is used to produce a number of clusters of points potentially containing the local and global maximum points. After that, the SPO algorithm is applied simultaneously to each cluster. Note that a cluster with center at $\mathbf{x}$ and radius of $\rho$ is the set of all points $\mathbf{y}$ satisfying $\|\mathbf{x}-\mathbf{y}\|<\rho$.

In step 1 of SPO algorithm in Section 2.1, an initial population of points is generated randomly. One difficulty with random numbers is that the obtainin $g$ numbers may fail to di stribute 'uniformly' in the sense of equidistributional (see [11]). As the consequence, the generated initial population of points may not uniformly distributed in the feasible region of the problem. It is $\mathrm{m}$ uch more desirable to have the uniform distribution of i nitial population of points in order to obtain as much as possible candi date solutions in the clustering phase. It will be helpful if the generated population of points in the search region has minimum deviation from uniformity (also called discre pancy). Various low discrepancy sequence of points have been constructed and S obol se quence is o ne of them. The useful ness of S obol sequence of points in finding $\mathrm{m}$ ultiple s olutions of syste $\mathrm{m}$ of nonli near equations in a single run have bee $\mathrm{n}$ shown in [1]. Considering the advantage of having low discrepancy sequence of points rather than pseudo-random sequence of points, we propose the u sage of Sobol sequence of points as init ial points $\mathbf{x}_{i}(0) \in \mathbb{R}^{n}$ both in step 1 of SPO algorithm and of 
Clustering phase. In this paper, we use the algorithm developed by Joe and Kuo [12]. This no-ra ndom technique will make this algorithm always give the same results from same input. Therefore we can concentrate to revise the parameter input without the need to repeatedly run the program in a large number of iterations.

Below we give more detail on the clustering technique.

Input : $m_{c l}, r_{c l}, \theta_{c l}, k_{c l}$ parameters for SPO algorithm at diversification phase

$\varepsilon(0<\varepsilon<1)$ parameter for optimum points acceptance

$\delta(0<\delta<1)$ parameter to distinguish between one candidate optimum and another one in case they are very close each other

$m, r, \theta, k_{\max } \quad$ parameters for SPO algorithm at intensification phase

\section{Process :}

Diversification phase

1. Generate $m_{c l}$ Sobol sequen ce of points as initial points $\quad \mathbf{x}_{i}(0) \in \mathbb{R}^{n}, i=1,2, \ldots, m_{c l}$ in the feasible regi on $D=\left[a_{1}, b_{1}\right] \times\left[a_{2}, b_{2}\right] \times \ldots \times\left[a_{n}, b_{n}\right] \subset \mathbb{R}^{n}$, and set $k=0$.

2. Set $\mathbf{x}^{*}=\mathbf{x}_{i g}(0), i_{g}=\arg \max _{i} F\left(\mathbf{x}_{i}(0)\right), i=1,2, \ldots, m_{c l}$ as center of the first cluste $\mathrm{r}$ with $\mathrm{r}$ adius e qual to $\frac{1}{2}\left(\min _{l}\left|b_{l}-a_{l}\right|\right), l=1,2, \ldots, n$.

3. For $i=1,2, \ldots, m_{c l}$ do :

If $\mathbf{x}_{i}$ is not the center of existing cluster, then do the function cluster below with $\mathbf{x}_{i}$ as input.

Function Cluster (input $\mathbf{y}$ )

a. Find a cluster with its center nearest to $\mathbf{y}$. Let $C$ be that cluster, with centre at $\mathbf{x}_{C}$.

b. Set $\mathbf{x}_{m}$ as mid-point between $\mathbf{y}$ and $\mathbf{x}_{C}$.

c. Compare $F(\mathbf{y}), F\left(\mathbf{x}_{C}\right)$, and $F\left(\mathbf{x}_{m}\right)$ :

- If $F\left(\mathbf{x}_{m}\right)<F(\mathbf{y})$ and $F\left(\mathbf{x}_{m}\right)<F\left(\mathbf{x}_{C}\right)$ : Set a new cluster with center at $\mathbf{y}$ and ra dius equal to the distance between $\mathbf{y}$ and $\mathbf{x}_{m}$.

- Else, if $F\left(\mathbf{x}_{m}\right)>F(\mathbf{y})$ and $F\left(\mathbf{x}_{m}\right)>F\left(\mathbf{x}_{C}\right)$ : Set a new cluster with $\mathbf{y}$ as its center and radius equal to the distance between $\mathbf{y}$ and $\mathbf{x}_{m}$. Redo Function Cluster with $\mathbf{x}_{m}$ as its input.

- Else, if $F(\mathbf{y})>F\left(\mathbf{x}_{c}\right)$, set $\mathbf{y}$ as the centre of $C$.

d. Change the radius of $C$ equal to the distance between $\mathbf{y}$ and $\mathbf{x}_{m}$.

4. Set $\mathbf{x}^{*}=\mathbf{x}_{i_{g}}$ where $i_{g}=\underset{i}{\arg \max } F\left(\mathbf{x}_{i}(k)\right), i=1,2, \ldots, m_{c l}$.

5. Update $\mathbf{x}_{i}: \mathbf{x}_{i}(k+1)=S_{n}\left(r_{c l}, \theta_{c l}\right) \mathbf{x}_{i}(k)-\left(S_{n}\left(r_{c l}, \theta_{c l}\right)-I_{n}\right) \mathbf{x}^{*}, i=1,2, \ldots, m_{c l}$, and set $k=k+1$.

6. Do steps 3 to $5 k_{c l}$ times

Intensification phase

7. Having done diversification phase, we obtain a number of clusters : $C_{1}, C_{2}, \ldots, C_{n_{C}}$. Each cluster has center at $\mathbf{x}_{C_{i}}$ an $\mathrm{d}$ ra dius of $\rho_{i}\left(i=1,2, \ldots, n_{C}\right)$. To e ach c luster, perform SPO algo rithm to obtain a can didate of maximum $\mathrm{p}$ oint in each cluster. Use $m, r, \theta, k_{\max }$ as SPO input in this phase, wit

$D_{i}=\left[x_{1, i}-\rho_{i}, x_{1, i}+\rho_{i}\right] \times\left[x_{2, i}-\rho_{i}, x_{2, i}+\rho_{i}\right] \times \cdots \times\left[x_{n, i}-\rho_{i}, x_{n, i}+\rho_{i}\right] \subset \mathbb{R}^{n}$ as $\mathrm{d}$ omain of the sea rch region where $\mathbf{x}_{C_{i}}=\left(x_{1, i}, x_{2, i}, \ldots, x_{n, i}\right)^{T} i=1,2, \ldots, n_{C}$.

Final selection

8. Keep only candidate maximum points which satisfy condition $F(\mathbf{x}-\varepsilon)<F(\mathbf{x})$ and $F(\mathbf{x}+\varepsilon)<F(\mathbf{x})$.

9. Suppose from step 8 there result $n_{g}$ candidate maximum points. From these $n_{g}$ candidates, select only those which satisfy $\left\|\mathbf{x}_{i}-\mathbf{y}_{j}\right\|>\delta \quad i, j=1,2, \ldots, n_{g}$ where $\left\|\mathbf{x}_{i}-\mathbf{x}_{j}\right\|$ is the distance between the candidates $\mathbf{x}_{i}$ and $\mathbf{x}_{j}$. In case where $\left\|\mathbf{x}_{i}-\mathbf{x}_{j}\right\| \leq \delta$, select only $\mathbf{x}_{i}$ as a maximum point if $F\left(\mathbf{x}_{i}\right) \geq F\left(\mathbf{x}_{j}\right)$, otherwise select $\mathbf{x}_{j}$ as a maximum point.

Output : all candidates from step 9 that meet final selection become the maximum points.

Note : to solve minimization problems, we run the same algorithm above with $G(\mathbf{x})=-F(\mathbf{x})$ as the objective function. 
In some cases, it is intended to obtain all of global optimum points only, without local optimum points. For this case, we do some modifications in the algorithm in section 2.2 to get global optimum only. See problems 4 and 5 in Section 3. The modifications are written below.

- Add $\gamma(0<\gamma<1)$ 'cut-off' parameter as additional input

- In step 3, add criteria $F\left(\mathbf{x}_{i}\right)>\gamma F\left(\mathbf{x}^{*}\right)$ before execute Function Cluster

- In step 8, add condition $F(\mathbf{x})>(1-\gamma) F\left(\mathbf{x}^{*}\right)$

\section{Numerical Experiments}

Several test cases from various problems, so called benchmark problems, have been examined to validate our proposed method. All num erical experiments were performed on a deskto $\mathrm{p}$ computer equipped with pr ocessor Intel $\mathrm{C}$ ore ${ }^{\mathrm{TM}} \mathrm{i} 5$ with $4 \mathrm{~GB}$ ram and 3.2 GHz CPU running Linux Mint 17.2. The code was written in $\mathrm{C}++$ and compiled using g++.

\subsection{Problem 1}

Consider the 2-dimensional second minima function as follows

$$
g(x, y)=\frac{1}{2}\left(x^{4}-16 x^{2}+5 x\right)+\frac{1}{2}\left(y^{4}-16 y^{2}+5 y\right) \text { where } D=\left\{(x, y)^{T} \in \mathbb{R}^{2} \mid-4 \leq x \leq 4,-4 \leq y \leq 4\right\}
$$

Using parameters $m_{c l}=300, r_{c l}=0.95, \theta_{c l}=\pi / 4, k_{c l}=10, \varepsilon=10^{-7}, \delta=0.1, m=200, \quad k_{\max }=200$, $r=0.95$, and $\theta=\pi / 4$, the results are obtained in $0.65 \mathrm{~s}$ and shown in Table 2 .

Table 2 : The optimum points for function (2)

\begin{tabular}{cccc|cccc}
\hline No & $x$ & $y$ & $g(x, y)$ & No & $x$ & $y$ & $g(x, y)$ \\
\hline \multicolumn{7}{c}{ Minimum Max } & \multicolumn{5}{c}{ imum } \\
1 & $-2.90353-$ & 2.90353 & -78.3323 & 1 & 0.1567310 & .1567310 & .391225 \\
2 & -2.903532 & $.7468-$ & 64.1956 & & & & \\
3 & 2.7468 & -2.90353 & -64.1956 & & & & \\
4 & 2.7468 & 2.7468 & -50.0589 & & & & \\
\hline
\end{tabular}

These optimum points can be confirmed by observing the graph in Figure 1 .

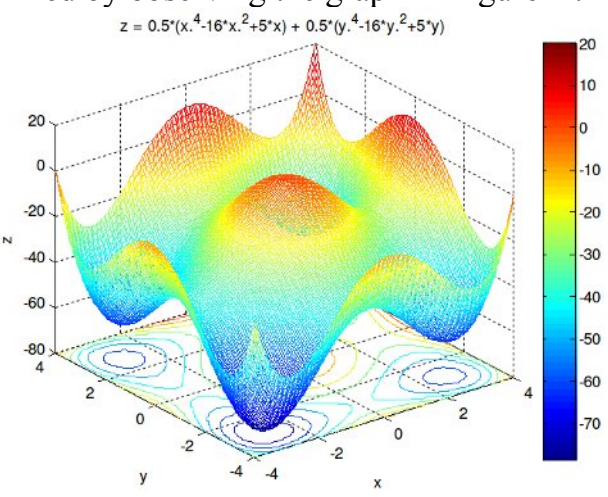

Figure 1: 2-dimensional second minima function

\subsection{Problem 2}

Consider the Six Hump Camel Back function as follows:

$$
g(x, y)=\left(4-2.1 x^{2}+\frac{x^{4}}{3}\right) x^{2}+x y+\left(4 y^{2}-4\right) y^{2}, \text { where } D=\{(x, y) \mid-1.9 \leq x \leq 1.9,-1.1 \leq y \leq 1.1\}
$$

Based on [10], it has two glo bal optima and two local o ptima. Using parameters $m_{c l}=1000, r_{c l}=0.99, \theta_{c l}=\pi / 2$, $k_{c l}=20, \varepsilon=10^{-5}, \delta=0.1, m=200, k_{\max }=200, r=0.95$, and $\theta=\pi / 4$, the results are obtained in 5.14s. They consist of 2 couples of global optima, which are 2 global maximum points and 2 global minimum points, and 2 couples of local optima, which are 4 local minimum points with 2 different values of $g(x, y)$.

Table 3: The optimum points for Six Hump Camel Back function (3)

\begin{tabular}{cccc|cccc}
\hline No & $x$ & $y$ & $g(x, y)$ & No & $x$ & $y$ & $g(x, y)$ \\
\hline \multicolumn{3}{c|}{ Minimum Max } & \multicolumn{4}{c}{ imum } \\
1 & -1.70361 & 0.796084 & -0.215464 & - & 1.23024 & -0.162328 & 2.4963 \\
\hline
\end{tabular}




\begin{tabular}{cccc|cccc}
\hline $2-$ & 1.6071 & -0.568651 & 2.10425 & 2 & 1.23023 & 0.162334 & 2.4963 \\
$3-$ & 0.089842 & 0.712656 & -1.03163 & & & & \\
40 & .089842 & -0.712656 & -1.03163 & & & & \\
51 & .6071 & 0.568651 & 2.10425 & & & & \\
61 & .70361 & -0.796084 & -0.215464 & & & & \\
\hline
\end{tabular}

\subsection{Problem 3}

Consider the $n$-dimensional Rastrigin function with our chosen domain as follows:

$$
g(\mathbf{x})=\sum_{i=1}^{n} x_{i}^{2}-10 \cos \left(2 \pi x_{i}\right)+10, \text { where } D=\left\{\mathbf{x}=\left(x_{1}, \ldots, x_{n}\right)^{T} \in \mathbb{R}^{n} \mid-1 \leq x_{i} \leq 1, i=1, \ldots, n\right\}
$$

For $n=2$, the $\mathrm{c}$ hosen parameters are $m_{c l}=500, r_{c l}=0.95, \theta_{c l}=\pi / 4, k_{c l}=10, \varepsilon=10^{-6}, \delta=0.1, m=200$, $k_{\max }=200, r=0.95$, and $\theta=\pi / 4$, and the results shown in Table 4 are obtained only in 0.53 s. It contains 4 global maximum points f or $g(\mathbf{x})=40.5025$, a gl obal minimum point, a nd 2 s ets co ntaining fo ur points of local $\mathrm{m}$ inimum points where $g(\mathbf{x})=0.994959$ and $g(\mathbf{x})=1.98992$ respectively.

Table 4: The optimum points for 2-dimensional Rastrigin function (4)

\begin{tabular}{|c|c|c|c|c|c|c|c|}
\hline No & $x_{1}$ & $x_{2}$ & $g(\mathbf{x})$ & No & $x_{1}$ & $x_{2}$ & $g(\mathbf{x})$ \\
\hline \multicolumn{4}{|c|}{ Minimum Max } & \multicolumn{4}{|c|}{ imum } \\
\hline $1-$ & 0.994959 & -0.994959 & 1.98992 & 1 & -0.502545 & -0.502546 & 40.5025 \\
\hline $2-$ & 0.994959 & $1.6366 \mathrm{e}-09$ & 0.994959 & 2 & -0.502537 & 0.502538 & 40.5025 \\
\hline $3-$ & 0.994959 & 0.994959 & 1.98992 & 3 & 0.502546 & -0.502545 & 40.5025 \\
\hline 41 & $.06667 \mathrm{e}-09$ & -0.994959 & 0.994959 & 4 & 0.502548 & 0.502544 & 40.5025 \\
\hline 50 & & 0 & 0 & & & & \\
\hline $6-$ & $5.10989 \mathrm{e}-09$ & 0.994959 & 0.994959 & & & & \\
\hline 70 & .994959 & -0.994959 & 1.98992 & & & & \\
\hline 80 & .994959 & $1.20281 \mathrm{e}-09$ & 0.994959 & & & & \\
\hline 90 & 994959 & 0.994959 & 1.98992 & & & & \\
\hline
\end{tabular}

For $n=3$, the chosen parameters are $m_{c l}=20000, r_{c l}=0.95, \theta_{c l}=\pi / 4, k_{c l}=20, \varepsilon=10^{-5}, \delta=0.1, m=200$, $k_{\max }=200, r=0.95$, and $\theta=\pi / 4$, and the results shown in Table 5 are obtained in $40.39 \mathrm{~s}$. It consists of 8 global maximum points, a global minimum point, and 3 sets of local minimum points containing 1, 5 and 5 points respectively. Table 5: The optimum points for 3-dimensional Rastrigin function (4)

\begin{tabular}{|c|c|c|c|c|c|c|c|c|c|}
\hline No & $x_{1}$ & $x_{2}$ & $x_{3}$ & $g(\mathbf{x})$ & No & $x_{1}$ & $x_{2}$ & $x_{3}$ & $g(\mathbf{x})$ \\
\hline & \multicolumn{4}{|c|}{ Minimum Max } & \multicolumn{5}{|c|}{ imum } \\
\hline 1 & -0.994957 & -0.99496 & -0.994959 & 2.98488 & 1 & $-0.502544-$ & $0.502554-$ & 0.5025416 & 0.7538 \\
\hline 2 & -0.994959 & $3.25509 \mathrm{e}-06$ & $-1.10394 \mathrm{e}-06$ & 0.9949592 & & 0.502514 & -0.502551 & 0.502528 & 60.7538 \\
\hline $3-$ & $4.14247 \mathrm{e}-07$ & -0.994957 & -0.99496 & 1.98992 & 3 & -0.502576 & 0.502551 & -0.50251 & 60.7538 \\
\hline 4 & $-1.65449 \mathrm{e}-08$ & -0.994959 & $-2.49482 \mathrm{e}-08$ & 0.9949594 & & 0.502537 & 0.502541 & 0.502545 & 60.7538 \\
\hline 5 & $6.19158 \mathrm{e}-07$ & -0.994959 & 0.994959 & 1.98992 & 5 & 0.502525 & $-0.502547-$ & 0.5025296 & 0.7538 \\
\hline 6 & $-2.2665 e-08$ & $3.28811 \mathrm{e}-08$ & -0.994959 & 0.994959 & 6 & $0.502574-$ & 0.502550 & .502525 & 60.7538 \\
\hline 7 & 000 & & & 0 & 7 & 0.502526 & 0.502573 & -0.502552 & 60.7538 \\
\hline 82 & $.12297 \mathrm{e}-06$ & 0.994959 & -0.994958 & 1.98992 & 80 & .502563 & 0.502542 & 0.502535 & 60.7538 \\
\hline 9 & $4.95836 \mathrm{e}-08$ & 0.994959 & $1.7007 \mathrm{e}-08$ & 0.994959 & & & & & \\
\hline 10 & $-4.1896 \mathrm{e}-080$ & .994956 & 0.9949571 & 98992 & & & & & \\
\hline 11 & 0.994959 & $-4.88344 \mathrm{e}-08$ & $2.27944 \mathrm{e}-08$ & 0.994959 & & & & & \\
\hline 12 & 0.994959 & 0.994959 & $1.72968 \mathrm{e}-06$ & 1.98992 & & & & & \\
\hline
\end{tabular}

\subsection{Problem 4}

Consider the 2-dimensional Vincent function as follows:

$$
g(x, y)=\frac{1}{2}(\sin (10 \log (x))+\sin (10 \log (\mathrm{y}))), \text { where } D=\left\{(x, y)^{T} \in \mathbb{R}^{2} \mid 0.25 \leq x \leq 10,0.25 \leq y \leq 10\right\}
$$

The f unction with the general dim ension $d$ in $[10] \mathrm{h}$ as $6^{d}$ glo bal o ptima and $\mathrm{n}$ o lo cal optim a. Using parameter $m_{c l}=1000, r_{c l}=0.95, \theta_{c l}=\pi / 4, k_{c l}=10, \varepsilon=10^{-5}, \delta=0.01, \gamma=0.2, m=150, k_{\max }=150, r=0.95$, and $\theta=\pi / 4$, we obtain $6^{2}=36$ maximum points in only $4.34 \mathrm{~s}$, which are shown in Table 6 . The maximum value $g(x, y)$ is 1 (one) for all points. 
Table 6: The optimum points for Vincent function (5)

\begin{tabular}{|c|c|c|c|c|c|c|c|c|c|c|c|}
\hline No & $x$ & $y$ & No & $x$ & $y$ & No & $x$ & $\bar{y}$ & No & $x$ & $y$ \\
\hline 1 & 0.3330180 & .333018 & 10 & 0.6242282 & .19328 & 19 & 2.193280 & 3330182 & 8 & 4.111212 & .19328 \\
\hline 2 & 0.3330180 & .624228 & 11 & 0.6242284 & . 11121 & 20 & 2.193280 & .6242282 & 9 & 4.111214 & .11121 \\
\hline 3 & 0.3330181 & .17009 & 12 & 0.6242287 & .70628 & 21 & 2.193281 & .17009 & 30 & 4.111217 & .70628 \\
\hline 4 & 0.3330184 & .11121 & 13 & 1.17009 & 0.333018 & 2 & 2.193282 & 19328 & 31 & 7.706280 & .333019 \\
\hline 5 & 0.3330184 & .11121 & 14 & 1.17009 & 0.624228 & 3 & 2.193284 & .11121 & 32 & 7.706280 & .624228 \\
\hline 6 & 0.333018 & 7.70628 & 15 & 1.170091 & 170092 & 4 & 2.19328 & 7.706273 & 3 & 7.70628 & 1.17009 \\
\hline 7 & 0.6242280 & .333018 & 16 & 1.17009 & 2.19328 & 25 & 4.111210 & .3330183 & 4 & 7.706282 & .19328 \\
\hline 8 & 0.6242280 & .624229 & 17 & 1.17009 & 4.11121 & 26 & 4.111210 & .6242293 & 5 & 7.706284 & .11121 \\
\hline 9 & 0.6242281 & .17009 & 18 & 1.17009 & 7.70628 & 27 & 4.111211 & .17009 & 36 & 7.706287 & .70628 \\
\hline
\end{tabular}

\subsection{Problem 5}

Consider the $n$-dimensional Shubert function as follows:

$$
g(\mathbf{x})=-\prod_{i=1}^{n} \sum_{j=1}^{5} j \cos \left[(j+1) x_{i}+j\right], \text { where } D=\left\{\mathbf{x}=\left(x_{1}, \ldots, x_{n}\right)^{T} \in \mathbb{R}^{n} \mid-10 \leq x_{i} \leq 10, i=1, \ldots, n\right\}
$$

In [10], the function has $n 3^{n}$ global optima and $\mathrm{m}$ any local optim a. For $n=2$, we choo se parameters $m_{c l}=10000$, $r_{c l}=0.95, \theta_{c l}=\pi / 4, k_{c l}=20, \varepsilon=10^{-6}, \delta=0.1, \gamma=0.5, m=200, k_{\max }=200, r=0.95$, and $\theta=\pi / 4$. There are 18 global maximum points with value 186.731 obtained only in $111.66 \mathrm{~s}$. The results are shown in Table 7.

Table 7: The maximum points of Shubert function (6) with $n=2$

\begin{tabular}{ccc|ccc|ccc}
\hline No & $x_{1}$ & $x_{2}$ & No & $x_{1}$ & $x_{2}$ & No & $x_{1}$ & $x_{2}$ \\
\hline $1-$ & 7.70831 & -7.08351 & & -1.42513 & -7.08351 & 13 & 4.85806 & -7.08351 \\
$2-$ & 7.70831 & -0.80032 & & -1.42513 & -0.800322 & 14 & 4.85806 & -0.80032 \\
3 & -7.70831 & 5.482869 & & -1.42513 & 5.482871 & 5 & 4.85806 & 5.48286 \\
4 & -7.08351 & -7.70831 & $10-$ & 0.800321 & -7.70831 & 165 & .48286 & -7.70831 \\
5 & -7.08351 & -1.42513 & $11-$ & 0.800323 & -1.42513 & 175 & .48286 & -1.42513 \\
6 & -7.08351 & 4.85806 & $12-$ & 0.800323 & 4.85806 & 185 & .48286 & 4.85806 \\
\hline
\end{tabular}

For $n=3$, we ch oose parameters $m_{c l}=50000, r_{c l}=0.99, \theta_{c l}=\pi / 4, k_{c l}=100, \varepsilon=10^{-2}, \delta=0.3, \gamma=0.5$, $m=300, k_{\max }=300, r=0.95$, a nd $\theta=\pi / 4$. They are 81 global maximum points with value 2709.09 only in $1468.63 \mathrm{~s}$.

\section{Conclusion}

Combination of the proposed clustering technique with SPO algo rithm and Sobol sequence of points have been shown able to obtain the maximum and minimum points, both local and global for the benchmark functions in a single run for each problem. Optimization of Vincent $n=2$ and Shubert $n=2,3$ functions gives the same numbers of global optima as in [10]. However, the result from Six Hump Camel Back function gives more points than in [10].

Acknowledgement: This work was partially supp orted by the P 3MI Industrial and Financial Mathematics Research Group, Institut Teknologi Bandung 2017.

\section{References}

[1] Sidarto, K.A., Kania, A.: Finding all soluti ons of sy stems of nonlinear equations using spiral dy namics inspired optimization with clustering. J. of Advanced Computational Intelligence and Intelligent Informatics (JACIII) 19(5), 697-707 (2015). DOI 10.20965/jaciii.2015.p0697

[2] Kamyab, S., Eftekhari, M.: Feature selection using $\mathrm{m}$ ultimodal optim ization tech niques. Neurocomputing, 171, 586-597 (2016). DOI 10.1016/j.neucom.2015.06.068

[3] Huang, L., Ng, C., Sheikh, A.H., Griffith, M.C.: Niching particle swarm optimization techniques for multimodal buckling $\mathrm{m}$ aximization o f com posite lam inates. Ap plied $\mathrm{S}$ oft $\mathrm{C}$ omputing, 57, 4 95-503 ( 2017). DOI 10.1016/j.asoc.2017.04.006

[4] Zaharie, D.: A multipopulation differential evolution algorithm for multimodal optimization. In: R. Matousek (ed.) Proceedings of 10th International Conference on Soft Computing - MENDEL 2004, no. 10 in MENDEL, pp. 1724. Brno University of Technology, VUT Press, Brno (2004)

[5] Chica, M ., B arranquero, J., Kajdanowicz, T., Damas, S., Cordón, O.: Multi modal optim ization: An effective framework for model calibration. Information Sciences, 375, 79-97 (2017). DOI 10.1016/j.ins.2016.09.048 
[6] Chiu, C., Lin, J.T.: N ovel hybrid approach with elite group optimal computing budget allocation for the stocha stic multimodal problem, Neurocomputing (2017). DOI 10.1016/j.neucom.2017.04.041

[7] Tamura, K., Yasuda, K.: Spiral Dynam ics Inspired Optimization. J. of Advanced Co mputational Intelligence and Intelligent Informatics (JACIII) 15(8), 1116-1122 (2011). DOI 10.20965/jaciii.2011.p1116.

[8] Tamura, K., Yasuda, K.: A Param eter Setting Method for Spiral Optimization from Stability Analysis of Dynamic Equilibrium Point. S ICE J ournal of C ontrol, M easurement, an d Sy stem Integrati on 7(3), 1 73-182 (2 014). DOI 10.9746/jcmsi.7.173

[9] Nasir, A.N.K, Ism ail, R .M.T.R., T okhi, M.O.: A daptive spiral dy namics metaheuristic alg orithm for gl obal optimisation with application to modelling of a flexible system. Applied Mathematical Modelling 40(9-10), 54425461 (2016). DOI 10.1016/j.apm.2016.01.002

[10]Li, X., Engelbrecht, A., Epitropakis, M.G.: Benchmarks Functions for CEC'2013 Special Session and Competition on Niching Methods for Multimodal Function Optimization. (Last updated 21 September 2016). Technical Report, Evolutionary Computation and Machine Learning Group, RMIT University, Australia.

[11] Seydel, R.: Tools for Computational Finance. Springer-Verlag (2002)

[12] Joe, S., Kuo, F.Y.: Constructing Sobol sequences with be tter two dimensional projections. SIAM J. Sci. Comput. 30(5), 2635-2654 (2008). DOI 10.1137/070709359 